\title{
Invariant criteria for the zero-coupon bond pricing Vasicek and Cox-Ingersoll-Ross Models
}

\author{
Ahmet Bakkaloglu ${ }^{1,2}$, Taha Aziz ${ }^{1}$ and F.M. Mahomed $^{3}$ \\ ${ }^{1}$ DST-NRF Centre of Excellence in Mathematical and Statistical Sciences, University of the Witwatersrand, Johannesburg,Wits \\ sl2050, South Africa \\ ${ }^{2}$ Department of Mathematics, Faculty of Arts and Sciences, Mimar Sinan Fine Arts University, Istanbul,Turkey \\ ${ }^{3}$ DST-NRF Centre of Excellence in Mathematical and Statistical Sciences, School of Computer Science and Applied \\ Mathematics,University of the Witwatersrand, Johannesburg,Wits s12050, South Africa
}

Received: 13 March 2017, Accepted: 25 April 2017

Published online: 26 April 2017.

\begin{abstract}
The zero coupon bond pricing Vasicek and Cox-Ingersoll-Ross (CIR) interest rate models are solved using the invariant approach. The invariance criteria is employed on the linear $(1+1)$ parabolic partial differential equations corresponding to the Vasicek and CIR models in order to perform reduction into one of the four Lie canonical forms. The invariant approach helps in transforming the partial differential equation representing the Vasicek model into the first Lie canonical form which is the classical heat equation. We also find that the invariant method aids in transforming the CIR model into the second Lie canonical form and with a proper parametric selection, the CIR equation can be converted to the first Lie canonical form. For both the Vasicek and CIR models, we obtain the transformations which map these equations into the heat equation and also to the second Lie canonical form. We construct the fundamental solutions for the Vasicek and CIR models via these transformations by utilizing the well-known fundamental solutions of the classical heat equation as well as solution to the second Lie canonical form. Finally, the closed-form analytical solutions of the Cauchy initial value problems of the Vasicek and CIR models with suitable choice of terminal boundary conditions are also deduced.
\end{abstract}

Keywords: Vasicek and CIR models, invariant approach, Lie symmetry, fundamental solutions, cauchy problem.

\section{Introduction}

Much literature on the applications of Lie symmetries to differential equations are to be found since the earliest works of Lie on the subject. In recent years, Lie group theoretic approach has been applied to differential equations from financial mathematics and economics. One of the initial works has been performed by Gazizov and Ibragimov [1], in which the authors have performed the symmetry analysis of the one-dimensional Black-Scholes equation. Goard [2] investigated the same problem again using Lie theory to unearth further insights of the Black-Scholes and related models. Lo and Hui in [3] utilized the Lie-algebraic approach for pricing moving barrier options with time-dependent parameters. Mahomed et al. [4] performed the complete classification of a bond pricing equation with the use of the invariant approach. Bakkaloğlu et al. [5] worked on optimal investment-consumption problem with CEV model by invariant approach. Pooe et al. [6], İzgi and Bakkaloğlu [7], [8], [9] investigated the fundamental solutions to the zero-coupon bond pricing equations with the Lie symmetry analysis. In recent times, the group approach has been widely applied to other partial differential equations of finance, for example, Naicker et al. [10], Ivanova et al. [11], Liu and Wang [12], Caister et al. [13], Sinkala [14] and an interesting topical review work by Hernández et al. [15].

The theory on bond-pricing models began in 1900 when the French mathematician Bachelier [16] deduced bond-pricing on the assumption that stock prices follow a Brownian motion. The works of Merton [17] and Black and Scholes [18] 
also opened a new era in the mathematical modelling of problems in finance. These models have been formulated in terms of stochastic differential equations. Moreover, the solution space of stochastic models have rich behavior. For example, İzgi investigates the behavioral classification of some stochastic models in mathematical finance in [19]. On the other hand, after certain assumptions these models may have been written in the form of linear parabolic partial differential equations (PDEs) with constant or variable coefficients. The classical bond-pricing equation was introduced by Vasicek [20], which was later modified by Cox et. al. [21], now known as the Cox-Ingersoll-Ross (CIR) Model.

A Lie symmetry group is used to construct symmetries of the underlying differential equations. Lie was the first who initiated the group classification of differential equations. Lie classified scalar linear second-order PDEs in two independent variables and developed a method for their integration. One of the earlier studies that proposed the invariant approach to Fokker-Planck equations was made by Johnpillai and Mahomed [22]. Semi-invariants for the $(1+1)$ linear parabolic equations with two independent variables and one dependent variable were derived in [22]. In addition, a joint invariant equation was obtained for the linear parabolic equation and the $(1+1)$ linear parabolic equation was reducible via a local equivalence transformation to the one- dimensional heat equation. They also proved necessary and sufficient conditions for a scalar linear $(1+1)$ parabolic equation, in terms of the coefficient of the equation, to be reducible via equivalence transformations to the classical heat equation. Later, Mahomed [23] gave the complete invariant characterization of a scalar linear $(1+1)$ parabolic partial differential equation of one space variable and one time variable into four canonical forms. In the last few years, the invariant approach has been successfully applied to some interesting linear $(1+1)$ parabolic PDEs $[4,24]$.

In this study, we investigate the scalar linear $(1+1)$ parabolic PDEs representing the classical Vasicek and Cox-Ingersoll-Ross (CIR) models from financial mathematics. We first briefly revisit the invariant criteria which can be found in [23]. We employed this approach to show that the parabolic PDEs which models the Vasicek CIR models are transformed into different Lie canonical forms. These transformations are further utilized to formulate the fundamental solutions of these models. Finally, the closed-form solutions of the Cauchy initial value problem for both the Vasicek and CIR models are also obtained.

\section{Vasicek and Cox-Ingersoll-Ross (CIR) bond pricing models}

A bond price is a long-term contract under which the borrower pays the bond holder a known amount on a fixed date at $t=T$, with $T$ the expiration time of the option. The bond contract includes periodic payments of cash dividends also, known as coupon, at fixed times during the life of the contract. If there is no coupon, the bond is known as a zerocoupon bond.

Suppose a zero-coupon bond yields $X$ dollars upon maturity at some future time say $T$. We denote $u(t, T)$, the price of the bond at time $t \leqslant T$. When $t=T$, the bond is exactly $X$ dollars, that is, $u(T, T)=X$. Now the question arise here is that how much it is worth when $t<T$. This is the main question of the zero-coupon bond-pricing problem. The bond price also depends on the rate of interest in the market. This is incorporated with some nondeterministic function $x(t)$, which is known as the instantaneous rate.

Two important models of the instantaneous rate which incorporate a number of empirically relevant characteristics of interest rates are the Vasicek model [15] and the Cox-Ingersoll-Ross (CIR) model [16]. In the Vasicek model it is assumed that the instantaneous rate $x(t)$ is a solution of the Ornstein-Uhlenbeck process of the form

$$
d x(t)=\kappa[\theta-x(t)] d t+\sigma d W(t)
$$


with $\kappa[\theta-x(t)]$ known as the drift term which represents the effect of pulling the interest rate towards its long-term mean with a magnitude $\theta$ proportional to the deviation of the interest rate from the mean. The parameter $\kappa$ determines the speed of the adjustment and $W(t)$ represents the standard Brownian motion.

On the other hand, in the CIR model, the instantaneous rate $x(t)$ is assumed to be governed by a stochastic process of the form

$$
d x(t)=\kappa[\theta-x(t)] d t+\sigma \sqrt{x(t)} d W(t) .
$$

The price of a zero coupon $u(x, t)$, in the Vasicek model, satisfies the parabolic PDE

$$
\frac{\partial u}{\partial t}+\frac{1}{2} \sigma^{2} \frac{\partial^{2} u}{\partial^{2} x}+\kappa(\theta-x) \frac{\partial u}{\partial x}-x u=0 .
$$

The CIR model satisfies the PDE given by

$$
\frac{\partial u}{\partial t}+\frac{1}{2} x \sigma^{2} \frac{\partial^{2} u}{\partial^{2} x}+\kappa(\theta-x) \frac{\partial u}{\partial x}-x u=0 .
$$

For both the Vasicek and CIR models, there is a terminal boundary condition, given by

$$
u(x, T)=1 .
$$

We here call the PDE (3) as the Vasicek equation and the PDE (4) the CIR equation.

\section{Invariant criteria for linear parabolic equations}

Here we give the main results of [18] on the invariant characterization of scalar linear $(1+1)$ parabolic PDEs.

The scalar linear $(1+1)$ parabolic PDE of one time and one space variable is given by

$$
\frac{\partial u}{\partial t}=a(t, x) \frac{\partial^{2} u}{\partial x^{2}}+b(t, x) \frac{\partial u}{\partial x}+c(t, x) u
$$

where $a, b$ and $c$ are the continuous functions of $t$ and $x$. The equivalence transformations [18] of the parabolic PDE (6) is an infinite group which comprises linear transformations of the dependent variable given by

$$
\bar{u}=\sigma(t, x) u, \sigma \neq 0
$$

and invertible transformations of independent variables

$$
\bar{t}=\phi(t), \bar{x}=\psi(t, x), \dot{\phi} \neq 0, \psi_{x} \neq 0
$$

where $\phi, \psi$ and $\sigma$ are arbitrary functions with restrictions for invertibility of the transformations and $\bar{u}$ is the new dependent variable. Two linear parabolic PDEs of the form (6) are equivalent to each other if one can be mapped to the other by appropriate combinations of the equivalence transformations (7) and (8). 
Lie proved that a scalar linear parabolic PDE (6) has the four canonical forms.

$$
\begin{aligned}
& \frac{\partial u}{\partial t}=\frac{\partial^{2} u}{\partial x^{2}}, \\
& \frac{\partial u}{\partial t}=\frac{\partial^{2} u}{\partial x^{2}}+\frac{A}{x^{2}} u, A \neq 0, \\
& \frac{\partial u}{\partial t}=\frac{\partial^{2} u}{\partial x^{2}}+c(x) u, c \neq 0, A / x^{2}, \\
& \frac{\partial u}{\partial t}=\frac{\partial^{2} u}{\partial x^{2}}+c(t, x) u, c \neq 0, A / x^{2} .
\end{aligned}
$$

The heat equation, which is the first Lie canonical form, has six nontrivial symmetries as well as the the infinite number of trivial superposition symmetries. The second Lie canonical form has four nontrivial symmetries. The third in general has two symmetries and the fourth has one nontrivial symmetry in general.

We now state the following theorems [18] which provide invariant criteria for the reduction of scalar linear $(1+1)$ parabolic PDE (6) into different Lie canonical forms given in Eq. (9).

Theorem 1. The scalar linear $(1+1)$ parabolic PDE (6) is reducible via equivalence transformations (7) and (8) to the heat equation

$$
\frac{\partial \bar{u}}{\partial \bar{t}}=\frac{\partial^{2} \bar{u}}{\partial \bar{x}^{2}}
$$

if and only if the coefficients of the parabolic PDE (6) satisfies the invariant condition

$$
2 L_{x}+2 M_{x}-N_{x}=0
$$

where

$$
L=|a|^{\frac{1}{2}}\left[|a|^{\frac{1}{2}} J_{x}\right]_{x}, M=|a|^{\frac{1}{2}}\left[|a|^{\frac{1}{2}} \partial_{t}(b / 2 a)\right]_{x} . N=|a|^{\frac{1}{2}} \partial_{t}^{2}\left(1 /|a|^{\frac{1}{2}}\right),
$$

with $J$ is

$$
J=c-\frac{b_{x}}{2}+\frac{b a_{x}}{2 a}+\frac{a_{x x}}{4}-\frac{3}{16} \frac{a_{x}^{2}}{a}-\frac{a_{t}}{2 a}-\frac{b^{2}}{4 a} .
$$

Theorem 2. The scalar linear (1+1) parabolic PDE (6) is reducible to the second Lie canonical form

$$
\frac{\partial \bar{u}}{\partial \bar{t}}=\frac{\partial^{2} \bar{u}}{\partial \bar{x}^{2}}+\frac{A}{\bar{x}^{2}} \bar{u}
$$

where $A$ is a nonzero constant, if and only if the coefficients of the parabolic PDE (6) satisfy the invariant equation, provided that condition (11) does not hold,

$$
\begin{aligned}
& 20 L_{x}+20 M_{x}-10 N_{x}+10\left[|a|^{\frac{1}{2}} M_{x}\right]_{x} \int \frac{d x}{|a|^{\frac{1}{2}}}-5\left[|a|^{\frac{1}{2}} N_{x}\right]_{x} \int \frac{d x}{|a|^{\frac{1}{2}}} \\
& +10\left[|a|^{\frac{1}{2}} L_{x}\right]_{x} \int \frac{d x}{|a|^{\frac{1}{2}}}+\left[|a|^{\frac{1}{2}}\left[|a|^{\frac{1}{2}} L_{x}\right]_{x}\right]_{x}\left(\int \frac{d x}{|a|^{\frac{1}{2}}}\right)^{2} \\
& +\left[|a|^{\frac{1}{2}}\left[|a|^{\frac{1}{2}} M_{x}\right]_{x}\right]_{x}\left(\int \frac{d x}{|a|^{\frac{1}{2}}}\right)^{2}-\frac{1}{2}\left[|a|^{\frac{1}{2}}\left[|a|^{\frac{1}{2}} N_{x}\right]_{x}\right]_{x}\left(\int \frac{d x}{|a|^{\frac{1}{2}}}\right)^{2}=0,
\end{aligned}
$$

where $L, M, N$ and $J$ are as given in (12) and (13). 
Theorem 3. The scalar linear (1+1) parabolic equation (6) which does not satisfy the conditions of Theorems 1 and 2 is equivalent to the third Lie canonical form

$$
\frac{\partial \bar{u}}{\partial \bar{t}}=\frac{\partial^{2} \bar{u}}{\partial \bar{x}^{2}}+\bar{c}(\bar{x}) \bar{u}
$$

if and only if the coefficients of parabolic equation (6) satisfy the invariant criterion, provided that the conditions (11) and (15) do not apply,

$$
\frac{\partial}{\partial t}\left[J+\frac{\partial}{\partial t} \int \frac{b}{2 a} d x-\frac{1}{2} \int \frac{1}{|a|^{\frac{1}{2}}}\left(\frac{\partial^{2}}{\partial t^{2}} \int \frac{d x}{|a|^{\frac{1}{2}}}\right) d x\right]=0
$$

Theorem 4. The scalar linear (1+1) parabolic equation (6) is equivalent to the fourth Lie canonical form

$$
\frac{\partial \bar{u}}{\partial \bar{t}}=\frac{\partial^{2} \bar{u}}{\partial \bar{x}^{2}}+\bar{c}(\bar{x}, \bar{t}) \bar{u}
$$

if and only if the coefficients of PDE (6) satisfy the invariant condition, provided that the conditions (11) and (14) do not hold,

$$
\frac{\partial}{\partial t}\left[J+\frac{\partial}{\partial t} \int \frac{b}{2 a} d x-\frac{1}{2} \int \frac{1}{|a|^{\frac{1}{2}}}\left(\frac{\partial^{2}}{\partial t^{2}} \int \frac{d x}{|a|^{\frac{1}{2}}}\right) d x\right] \neq 0
$$

Theorem 5. The linear parabolic equation (6) is reducible to the classical heat PDE (10) via the transformations

$$
\begin{aligned}
\bar{t} & =\phi(t) \\
\bar{x} & = \pm \int\left[\dot{\phi} a(t, x)^{-1}\right]^{\frac{1}{2}} d x+\beta(t) \\
\bar{u} & =v(t)[a(t, x)]^{-\frac{1}{4}} u \exp \left[\int \frac{b(t, x)}{2 a(t, x)} d x-\frac{1}{8} \frac{\ddot{\phi}}{\dot{\phi}}\left(\int \frac{d x}{a(t, x)^{\frac{1}{2}}}\right)^{2}\right. \\
& \left.-\frac{1}{2} \int \frac{1}{a(t, x)^{\frac{1}{2}}} \frac{\partial}{\partial t}\left(\int \frac{d x}{a(t, x)^{\frac{1}{2}}}\right) d x \pm \frac{1}{2} \frac{\dot{\beta}}{\dot{\phi}^{\frac{1}{2}}} \int \frac{d x}{a(t, x)^{\frac{1}{2}}}\right] .
\end{aligned}
$$

where $\dot{\phi}$ and a have the same sign, and $\phi, \beta$ and $v$ satisfy

$$
\begin{aligned}
\dot{\phi} \bar{c} & =J+\frac{\partial}{\partial t} \int \frac{b(t, x)}{2 a(t, x)} d x-\frac{1}{2} \int \frac{1}{a(t, x)^{\frac{1}{2}}} \frac{\partial^{2}}{\partial t^{2}}\left(\int \frac{d x}{a(t, x)^{\frac{1}{2}}}\right) d x \\
& +f(t)\left(\int \frac{d x}{a(t, x)^{\frac{1}{2}}}\right)^{2}+g(t)\left(\int \frac{d x}{a(t, x)^{\frac{1}{2}}}\right)+h(t),
\end{aligned}
$$

in which $J$ is as in Eq. (13) and

$$
\begin{aligned}
& f(t)=\frac{1}{16} \frac{\ddot{\phi}^{2}}{\dot{\phi}^{2}}-\frac{1}{8}\left(\frac{\ddot{\phi}}{\dot{\phi}}\right)_{t}, \\
& g(t)= \pm \frac{1}{4} \frac{\ddot{\phi}}{\dot{\phi}} \frac{\dot{\beta}}{\dot{\phi}^{\frac{1}{2}}} \pm \frac{1}{2}\left(\frac{\dot{\beta}}{\dot{\phi}^{\frac{1}{2}}}\right)_{t}, \\
& h(t)=\frac{1}{4} \frac{\ddot{\phi}}{\dot{\phi}}+\frac{1}{4} \frac{\dot{\beta}^{2}}{\dot{\phi}}+\frac{\dot{v}}{v}
\end{aligned}
$$

The functions $f, g$ and h are constrained by the relation defined in Eq. (21). For reductions into the other Lie canonical forms, the transformation relations are derived in [18]. 


\section{Invariant solutions of the Vasicek equation}

In this section, we apply the above theorems to the Vasicek PDE (3). We use the invariant approach encapsulated in the theorems above to simply and elegantly map the PDE (3) to their Lie canonical forms.

\subsection{Reduction to the heat equation}

With the comparison of Vasicek PDE (3) to the scalar linear (1+1) parabolic PDE (6), the coefficients $a(t, x), b(t, x)$ and $c(t, x)$ are written as

$$
\begin{aligned}
& a(t, x)=-\frac{1}{2} \sigma^{2}, \\
& b(t, x)=-\kappa(\theta-x), \\
& c(t, x)=x .
\end{aligned}
$$

Since the coefficients of the bond price PDE (3) are independent of $t$, so we have $M=N=0$. Therefore it is easy to evaluate $J$ as given in Eq. (13) for the Vasicek equation (3). Indeed it is

$$
J=x-\frac{\kappa}{2}+\frac{\kappa^{2}}{2 \sigma^{2}}(\theta-x)^{2} .
$$

The invariant condition (11) for reduction to the heat equation becomes

$$
L_{x}=0
$$

Thus obviously the invariant condition (11) is identically satisfied and we get reduction of the Vasicek PDE (3) to the heat equation.

\subsection{Transformation formulae and fundamental solutions}

We now find the transformations which reduce the PDE (3) to the heat equation. These transformations are further utilized to obtain the fundamental solutions of the Vasicek PDE (3).

Making use of the coefficients defined in Eq. (23) in Eqs. (21) and (22) in Theorem 5, we find

$$
\begin{aligned}
f(t) & =-\frac{\kappa^{2}}{4} \\
g(t) & =\frac{\kappa^{2} \theta}{\sqrt{2} \sigma}-\frac{\sigma}{\sqrt{2}}, \\
h(t) & =\frac{\kappa}{2}-\frac{\kappa^{2} \theta^{2}}{2 \sigma^{2}} \\
\phi(t) & =c_{1} \sqrt{\frac{2}{A}} \tan \left(\sqrt{\frac{A}{2}} t\right), \\
\beta(t) & =\frac{2 \sqrt{c_{1}} B}{A \cos \left(\sqrt{\frac{A}{2}} t\right)} .
\end{aligned}
$$


with $c_{1}, A=-8 f(t)$ and $B=g(t) / 2$ the constants of integration. With the use of the values of the functions from Eq. (25) into Eq. (20), the transformations that reduce the parabolic equation (3) into the heat equation are

$$
\begin{aligned}
& \bar{t}=c_{1} \sqrt{\frac{2}{A}} \tan \left(\sqrt{\frac{A}{2}} t\right) \\
& \bar{x}= \pm \frac{\sqrt{2 c_{1}}}{\sigma \cos \left(\sqrt{\frac{A}{2}} t\right)} x+\frac{2 \sqrt{c_{1}} B}{A \cos \left(\sqrt{\frac{A}{2}} t\right)}+c_{2} \\
& \bar{u}=\left(\frac{1}{2} \sigma^{2}\right)^{-\frac{1}{4}} \exp \left[\frac{1}{2} \ln \cos \left(\sqrt{\frac{A}{2}} t\right)-\frac{B^{2} \sqrt{2}}{2 A^{\frac{3}{2}}} \tan \left(\sqrt{\frac{A}{2}} t\right)+\frac{B^{2}}{2 A} t+c_{3}\right] u \\
& \exp \left[-\frac{\kappa}{2 \sigma^{2}}(\theta-x)^{2}-\frac{1}{4 \sigma^{2}}\left(\sqrt{2 A} \tan \left(\sqrt{\frac{A}{2}} t\right)\right) x^{2} \pm \frac{2 c_{2}}{\sigma \sqrt{A}} \tan \left(\sqrt{\frac{A}{2}} t\right) x+c_{4}\right],
\end{aligned}
$$

where $c_{i}(i=1, \ldots, 4)$ are the constants of integration. The transformed terminal condition for Vasicek equation is written as

$$
\begin{gathered}
\bar{u}(x, T)=\exp \left[-\frac{\kappa}{2 \sigma^{2}}(\theta-x)^{2}-\frac{1}{4 \sigma^{2}}\left(\sqrt{2 A} \tan \left(\sqrt{\frac{A}{2}} T\right)\right) x^{2} \pm \frac{2 B}{\sigma \sqrt{A}} \tan \left(\sqrt{\frac{A}{2}} T\right) x+c_{4}\right], \\
\left(\frac{1}{2} \sigma^{2}\right)^{-\frac{1}{4}} \exp \left[\frac{1}{2} \ln \cos \left(\sqrt{\frac{A}{2}} T\right)-\frac{B^{2} \sqrt{2}}{2 A^{\frac{3}{2}}} \tan \left(\sqrt{\frac{A}{2}} T\right)+\frac{B^{2}}{2 A} T+c_{3}\right] .
\end{gathered}
$$

We now construct the fundamental solutions of the Vasicek PDE (3). We have seen that there exist transformations (26) that reduce the PDE (3) to the classical heat equation. This result is used in the construction of the fundamental solution of the Vasicek equation.

The fundamental solution of the heat equation is well-known and is given in barred coordinates in [6]

$$
\bar{u}=\frac{1}{2 \sqrt{\bar{t} \pi}} \exp \left[-\frac{\bar{x}^{2}}{4 \bar{t}}\right] .
$$

We seek the fundamental solution for the PDE (3). The solution (28) is transformed by means of (26). Making use of Eqs. (28) and (29), the solution $u(x, t)$ is written as

$$
\begin{aligned}
& u(x, t)=\left(\frac{1}{2} \sigma^{2}\right)^{\frac{1}{4}} \frac{1}{2 \sqrt{\bar{t} \pi}} \exp \left[-\frac{\bar{x}^{2}}{4 \bar{t}}\right] \\
& \exp \left[-\left\{-\frac{\kappa}{2 \sigma^{2}}(\theta-x)^{2}-\frac{1}{4 \sigma^{2}}\left(\sqrt{2 A} \tan \left(\sqrt{\frac{A}{2}} t\right)\right) x^{2} \pm \frac{2 B}{\sigma \sqrt{A}} \tan \left(\sqrt{\frac{A}{2}} t\right) x+c_{4}\right\}\right] \\
& \exp \left[-\left\{\frac{1}{2} \ln \cos \left(\sqrt{\frac{A}{2}} t\right)-\frac{B^{2}}{\sqrt{2} A^{\frac{3}{2}}} \tan \left(\sqrt{\frac{A}{2}} t\right)+\frac{B^{2}}{2 A} t+c_{3}\right\}\right] .
\end{aligned}
$$


Finally with substitution of the values of $\bar{t}$ and $\bar{x}$ from Eq. (26) into Eq. (29), the solution becomes

$$
\begin{aligned}
& u(x, t)=\left(\frac{1}{2} \sigma^{2}\right)^{\frac{1}{4}} \frac{1}{2 \sqrt{c_{1} \sqrt{\frac{2}{A}} \tan \left(\sqrt{\frac{A}{2}} t\right) \pi}} \exp \left[-\left\{\frac{1}{2} \ln \cos \left(\sqrt{\frac{A}{2}} t\right)-\frac{B^{2}}{\sqrt{2} A^{\frac{3}{2}}} \tan \left(\sqrt{\frac{A}{2}} t\right)+\frac{B^{2}}{2 A} t+c_{3}\right\}\right] \\
& \exp \left[-\left\{-\frac{\kappa}{2 \sigma^{2}}(\theta-x)^{2}-\frac{1}{4 \sigma^{2}}\left(\sqrt{2 A} \tan \left(\sqrt{\frac{A}{2}} t\right)\right) x^{2} \pm \frac{2 B}{\sigma \sqrt{A}} \tan \left(\sqrt{\frac{A}{2}} t\right) x+c_{4}\right\}\right] \\
& \exp \left[-\frac{\left( \pm \frac{\sqrt{2 c_{1}}}{\sigma \cos \left(\sqrt{\frac{A}{2}} t\right)} x+\frac{2 \sqrt{c_{1}} B}{A \cos \left(\sqrt{\frac{A}{2}} t\right)}+c_{3}\right)^{2}}{4 c_{1} \sqrt{\frac{2}{A}} \tan \left(\sqrt{\frac{A}{2}} t\right)}\right] \text {. }
\end{aligned}
$$

Now we look for another form of solution for the Vasicek PDE (3) under the same set of transformation as for the heat equation. Another form of solution in series of the heat equation [25]

$$
\bar{u}=\sum_{n=1}^{\infty} b_{n} \exp \left[-\left(\frac{n \pi}{l}\right)^{2} k \bar{t}\right] \sin \left(\frac{n \pi \bar{x}}{l}\right),
$$

which converges uniformly for $0 \leq x \leq l, t \geq 0$. The solution (31) is transformed by means of transformation (26) and is given by

$$
\begin{aligned}
& u(x, t)=\left(\frac{1}{2} \sigma^{2}\right)^{\frac{1}{4}} \sum_{n=1}^{\infty} b_{n} \exp \left[-\left(\frac{n \pi}{l}\right)^{2} k \bar{t}\right] \sin \left(\frac{n \pi \bar{x}}{l}\right) \\
& \exp \left[-\left\{-\frac{\kappa}{2 \sigma^{2}}(\theta-x)^{2}-\frac{1}{4 \sigma^{2}}\left(\sqrt{2} A \tan \left(\sqrt{\frac{A}{2}} t\right)\right) x^{2} \pm \frac{2 B}{\sigma \sqrt{A}} \tan \left(\sqrt{\frac{A}{2}} t\right) x+c_{4}\right\}\right] \\
& \exp \left[-\left\{\frac{1}{2} \ln \cos \left(\sqrt{\frac{A}{2}} t\right)-\frac{B^{2}}{\sqrt{2} A^{\frac{3}{2}}} \tan \left(\sqrt{\frac{A}{2}} t\right)+\frac{B^{2}}{2 A} t+c_{3}\right\}\right] .
\end{aligned}
$$

Finally by making use of the values of $\bar{t}$ and $\bar{x}$ from (26), the solution $u(x, t)$ in Eq. (32) takes the form

$$
\begin{aligned}
u(x, t) & =\left(\frac{1}{2} \sigma^{2}\right)^{\frac{1}{4}} \exp \left[-\left\{\frac{1}{2} \ln \cos \left(\sqrt{\frac{A}{2}} t\right)-\frac{B^{2} \sqrt{2}}{2 A^{\frac{3}{2}}} \tan \left(\sqrt{\frac{A}{2}} t\right)+\frac{B^{2}}{2 A} t+c_{3}\right\}\right] \\
& \exp \left[-\left\{-\frac{\kappa}{2 \sigma^{2}}(\theta-x)^{2}-\frac{1}{4 \sigma^{2}}\left(\sqrt{2 A} \tan \left(\sqrt{\frac{A}{2}} t\right)\right) x^{2} \pm \frac{2 B}{\sigma \sqrt{A}} \tan \left(\sqrt{\left.\left.\left.\frac{A}{2} t\right) x+c_{4}\right\}\right]}\right.\right.\right. \\
& \sum_{n=1}^{\infty} b_{n} \exp \left[-\left(\frac{n \pi}{l}\right)^{2} k c_{1} \sqrt{\frac{2}{A}} \tan \left(\sqrt{\frac{A}{2}} t\right)\right] \sum_{n=1}^{\infty} \sin \left(\frac{n \pi\left( \pm \frac{\sqrt{2 c_{1}}}{\sigma \cos \left(\sqrt{\frac{A}{2}} t\right)} x+\frac{2 \sqrt{c_{1}} B}{A \cos \left(\sqrt{\frac{A}{2}} t\right)}+c_{2}\right)}{l}\right) .
\end{aligned}
$$




\subsection{Exact solution via the Cauchy problem of the heat equation}

In this section, we derive the closed-form analytical solution of the Cauchy problem (3) corresponding to the boundary condition (5) for the Vasicek model. We make use of the appropriate results on the Cauchy problem for classical heat equation to obtain the solution to our problem.

In the previous section, we have found the equivalence transformations which map the Vasicek equation (3) into the classical heat equation. These transformations are derived in Eq. (26) which reduce the Cauchy problem (3) subject to (5) for the Vasicek model into the standard heat Cauchy problem, given by

$$
\begin{aligned}
\frac{\partial \bar{u}}{\partial \bar{t}} & =\frac{\partial^{2} \bar{u}}{\partial \bar{x}^{2}}, \\
\bar{u}(\bar{x}, T) & =\phi(\bar{x}),
\end{aligned}
$$

for some function $\phi$ provided $\phi$ is "well-behaved". The solution to (34) and (35) is well-known [26] and is given by

$$
\bar{u}(\bar{x}, \bar{t})=\frac{1}{2 \sqrt{\bar{\tau} \pi}} \int_{-\infty}^{+\infty} \phi(\xi) \exp \left[-\frac{(\bar{x}-\zeta)^{2}}{4 \bar{t}}\right] d \xi .
$$

We need to transform the solution (36) into the solution of (3). Substituting $\bar{u}(\bar{x}, \bar{t})$ from Eq. (36) into Eq. (26), we obtain

$$
\begin{aligned}
& u(x, t)=\left(\frac{1}{2} \sigma^{2}\right)^{\frac{1}{4}} \int_{-\infty}^{+\infty} \phi(\xi) \exp \left[-\frac{(\bar{x}-\xi)^{2}}{4 \bar{t}}\right] d \xi \\
& \exp \left[-\left\{-\frac{\kappa}{2 \sigma^{2}}(\theta-x)^{2}-\frac{1}{4 \sigma^{2}}\left(\sqrt{2 A} \tan \left(\sqrt{\frac{A}{2}} t\right)\right) x^{2} \pm \frac{2 B}{\sigma \sqrt{A}} \tan \left(\sqrt{\frac{A}{2}} t\right) x+c_{4}\right\}\right] \\
& \exp \left[-\left\{\frac{1}{2} \ln \cos \left(\sqrt{\frac{A}{2}} t\right)-\frac{B^{2}}{\sqrt{2} A^{\frac{3}{2}}} \tan \left(\sqrt{\frac{A}{2}} t\right)+\frac{B^{2}}{2 A} t+c_{3}\right\}\right]
\end{aligned}
$$

where $\phi(\xi)$ is found from Eq. (26) By keeping in mind the boundary condition (27), we have

$$
\begin{gathered}
\phi(\xi)=\exp \left[-\frac{\kappa}{2 \sigma^{2}}(\theta-x)^{2}-\frac{1}{4 \sigma^{2}}\left(\sqrt{2 A} \tan \left(\sqrt{\frac{A}{2}} T\right)\right) x^{2} \pm \frac{2 B}{\sigma \sqrt{A}} \tan \left(\sqrt{\frac{A}{2}} T\right) x+c_{4}\right] \\
\left(\frac{1}{2} \sigma^{2}\right)^{-\frac{1}{4}} \exp \left[\frac{1}{2} \ln \cos \left(\sqrt{\frac{A}{2}} T\right)-\frac{B^{2}}{\sqrt{2} A^{\frac{3}{2}}} \tan \left(\sqrt{\frac{A}{2}} T\right)+\frac{B^{2}}{2 A} T+c_{3}\right] .
\end{gathered}
$$

Finally, using the values of $\bar{x}$ and $\bar{t}$ from Eq. (26) into Eq. (37), the solution $u(x, t)$ takes the form

$$
\begin{aligned}
u(x, t)=\left(\frac{1}{2} \sigma^{2}\right)^{\frac{1}{4}} \int_{-\infty}^{+\infty} \phi(\xi) \exp \left[-\frac{\left.\left( \pm \frac{\sqrt{2 c_{1}}}{\sigma \cos \left(\sqrt{\frac{A}{2}} t\right)} x+\frac{2 \sqrt{c_{1} B}}{A \cos \left(\sqrt{\frac{A}{2}} t\right)}+c_{3}-\xi\right)^{2}\right]}{4 c_{1} \sqrt{\frac{2}{A}} \tan \left(\sqrt{\frac{A}{2}} t\right)}\right] \xi \\
\exp \left[-\left\{-\frac{\kappa}{2 \sigma^{2}}(\theta-x)^{2}-\frac{1}{4 \sigma^{2}}\left(\sqrt{2 A} \tan \left(\sqrt{\frac{A}{2}} t\right)\right) x^{2} \pm \frac{2 B}{\sigma \sqrt{A}} \tan \left(\sqrt{\frac{A}{2}} t\right) x+c_{4}\right\}\right] \\
\exp \left[-\left\{\frac{1}{2} \ln \cos \left(\sqrt{\frac{A}{2}} t\right)-\frac{B^{2}}{\sqrt{2} A^{\frac{3}{2}}} \tan \left(\sqrt{\frac{A}{2}} t\right)+\frac{B^{2}}{2 A} t+c_{3}\right\}\right],
\end{aligned}
$$


with $\phi(\xi)$ given in Eq. (38). One can deduce the value of the integral in Eq. (39) in terms of the Kummer confluent hypergeometric functions.

\section{Invariant solutions of the Cox-Ingersoll-Ross (CIR) equation}

In this section, we employ the invariant approach to obtain the closed-form solution of a parabolic PDE derived by CoxIngersoll-Ross and given in Eq. (4). The PDE (4) is known as the CIR equation in the literature.

\subsection{Reduction of the CIR equation to the heat equation}

We now use the theorem of the invariant approach to find the equivalence transformations which reduce the PDE (4) to the Lie canonical forms. Making comparison of PDE (4) to the scalar linear $(1+1)$ parabolic partial differential equation (6), the coefficients functions $a(t, x), b(t, x)$ and $c(t, x)$ are written as:

$$
\begin{aligned}
& a(t, x)=-\frac{1}{2} \sigma^{2} x, \\
& b(t, x)=-\kappa(\theta-x), \\
& c(t, x)=x
\end{aligned}
$$

Making use of Theorem 1, the values of the parameters for which the PDE (4) can be mapped to the heat equation are obtained. First we have to evaluate $J$ as given in Eq. (13) for the CIR equation (4). It is given by

$$
J=x-\frac{\kappa}{2}+\frac{\kappa(x-\theta)}{2 x}+\frac{3}{32} \frac{\sigma^{2}}{x}+\frac{\kappa^{2}(x-\theta)^{2}}{2 \sigma^{2} x} .
$$

Since the coefficients of the zero-coupon bond price PDE (4) are independent of $t, M$ and $N$ are zero in the conditions defined in Theorem 1. For the value of $J$ given in Eq. (41), the invariant condition (11) for reduction to the heat equation becomes

$$
L_{x}=-\frac{3}{8}\left(\sigma^{4}+2 \kappa \theta \sigma^{2}-2 \kappa^{2} \theta^{2}\right) x^{-3}
$$

From Eq. (42), we can deduce

$$
\sigma^{2}=\frac{-8 \kappa \theta \pm 4 \kappa \theta}{-3}
$$

which results in

$$
\sigma_{i}(i=1,2,3,4)=\left[-2 \sqrt{\frac{\kappa \theta}{3}}, 2 \sqrt{\frac{\kappa \theta}{3}},-2 \sqrt{\kappa \theta}, 2 \sqrt{\kappa \theta}\right]
$$

The values of $\sigma$ in Eq. (44) give the non-trivial cases for which the invariant condition (11) is satisfied which results in the reduction of the CIR equation (4) to the heat equation.

\subsection{Transformation formulae and fundamental solutions}

Here we compute the transformations which reduce the PDE (4) to the heat equation. These transformations are further utilized to obtain the fundamental solutions of the CIR equation (4). Making use of the coefficients defined in Eq. (40) in 
Eqs. (21) and (22) in Theorem 5, we have

$$
\begin{aligned}
& f(t)=-\frac{1}{8}\left(\sigma^{2}+\frac{\kappa^{2}}{2}\right) \\
& g(t)=0 \\
& h(t)=\frac{\kappa^{2} \theta}{\sigma^{2}} \\
& \phi(t)=c_{1} \sqrt{\frac{2}{A} \tan \left(\sqrt{\frac{A}{2}} t\right)} \\
& \beta(t)=c_{2} \int \frac{d t}{\cos \left(\sqrt{\frac{A}{2}} t\right) \ln \cos \left(\sqrt{\frac{A}{2}} t\right)} \\
& v(t)=\exp \left[\frac{1}{2} \ln \cos \left(\sqrt{\frac{A}{2}} t\right)-\frac{c_{3}^{2}}{4} \int \frac{d t}{\left[\ln \cos \left(\sqrt{\frac{A}{2}} t\right)\right]^{2}}+B t+c_{4}\right]
\end{aligned}
$$

with $c_{i}(i=1,2,3,4), A=-8 f(t)$ and $B=h(t)$ are the constants of integration and $\sigma$ takes one of the values given in Eq. (44). With the use of the values of the functions from Eq. (45) into Eq. (20) and simplification, the transformations that reduce the parabolic equation (4) into the heat equation are obtained as follows

$$
\begin{aligned}
& \bar{t}=c_{1} \sqrt{\frac{2}{A}} \tan \left(\sqrt{\frac{A}{2}} t\right), \\
& \bar{x}= \pm \frac{2 \sqrt{2 c_{1}}}{\sigma \cos \left(\sqrt{\frac{A}{2}} t\right)} \sqrt{x}+c_{2} \int_{t}^{T} \frac{d t}{\cos \left(\sqrt{\frac{A}{2}} t\right) \ln \cos \left(\sqrt{\frac{A}{2}} t\right)}+c_{3} \text {, } \\
& \bar{u}(x, t)=\left(\frac{1}{2} \sigma^{2} x\right)^{-\frac{1}{4}} \exp \left[\frac{1}{2} \ln \cos \left(\sqrt{\frac{A}{2}} t\right)-\frac{c_{3}^{2}}{4} \int_{t}^{T} \frac{d t}{\left[\ln \cos \left(\sqrt{\frac{A}{2}} t\right)\right]^{2}}+B t+c_{4}\right] \\
& u \exp \left[\frac{\kappa}{\sigma^{2}}(\theta \ln x-x)-\frac{\sqrt{2 A}}{\sigma^{2}} \tan \left(\sqrt{\frac{A}{2}} t\right) x \pm \frac{\sqrt{2} c_{3}}{\sigma \ln \cos \left(\sqrt{\frac{A}{2}} t\right)} \sqrt{x}\right]
\end{aligned}
$$

where $c_{i}(i=1, \ldots, 4)$ are the constants of integration. The transformed terminal condition for the CIR equation is written as:

$$
\begin{aligned}
\bar{u}(x, T) & =\left(\frac{1}{2} \sigma^{2} x\right)^{-\frac{1}{4}} \exp \left[\frac{1}{2} \ln \cos \left(\sqrt{\frac{A}{2}} T\right)+B T+c_{4}\right] \\
& \exp \left\{\frac{\kappa}{\sigma^{2}}(\theta \ln x-x)-\frac{\sqrt{2 A}}{\sigma^{2}} \tan \left(\sqrt{\frac{A}{2}} T\right) x \pm \frac{\sqrt{2} c_{3}}{\sigma \ln \cos \left(\sqrt{\frac{A}{2}} T\right)} \sqrt{x}\right\} .
\end{aligned}
$$


Following the same methodology as adopted previously for the Vasicek model, the fundamental solutions of the CIR equation (4) for the case of reduction into the heat equation under the transformations (46) are given by

$$
\begin{aligned}
& u(x, t)=\frac{1}{2 \sqrt{c_{1} \sqrt{\frac{2}{A}} \tan \left(\sqrt{\frac{A}{2}} t\right) \pi}}\left(\frac{1}{2} \sigma^{2} x\right)^{\frac{1}{4}} \\
& \exp \left[-\left\{\frac{1}{2} \ln \cos \left(\sqrt{\frac{A}{2}} t\right)-\frac{c_{3}^{2}}{4} \int_{t}^{T} \frac{d t}{\left[\ln \cos \left(\sqrt{\frac{A}{2}} t\right)\right]^{2}}+B t+c_{4}\right\}\right] \\
& \exp \left[-\left\{\frac{\kappa}{\sigma^{2}}(\theta \ln x-x)-\frac{\sqrt{2 A}}{\sigma^{2}} \tan \left(\sqrt{\frac{A}{2}} t\right) x \pm \frac{\sqrt{2} c_{3}}{\sigma \ln \cos \left(\sqrt{\frac{A}{2}} t\right)} \sqrt{x}\right\}\right], \\
& \exp \left[-\frac{\left.\left( \pm \frac{2 \sqrt{2 c_{1}}}{\sigma \cos \left(\sqrt{\frac{A}{2}} t\right)} \sqrt{x}+c_{2} \int_{t}^{T} \frac{d t}{\cos \left(\sqrt{\frac{A}{2}} t\right) \ln \cos \left(\sqrt{\frac{A}{2}} t\right)}+c_{3}\right)^{2}\right]}{4 c_{1} \sqrt{\frac{2}{A}} \tan \left(\sqrt{\frac{A}{2}} t\right)}\right]
\end{aligned}
$$

and

$$
\begin{aligned}
& u(x, t)=\left(\frac{1}{2} \sigma^{2} x\right)^{\frac{1}{4}} \sum_{n=1}^{\infty} \sin \left(\frac{n \pi\left( \pm \frac{2 \sqrt{2 c_{1}}}{\sigma \cos \left(\sqrt{\frac{A}{2}} t\right)} \sqrt{x}+c_{2} \int_{t}^{T} \frac{d t}{\cos \left(\sqrt{\frac{A}{2}} t\right) \ln \cos \left(\sqrt{\frac{A}{2}} t\right)}+c_{3}\right)}{l}\right) \\
& \sum_{n=1}^{\infty} b_{n} \exp \left[-\left(\frac{n \pi}{l}\right)^{2} k c_{1} \sqrt{\frac{2}{A}} \tan \left(\sqrt{\frac{A}{2} t}\right)\right] \\
& \exp \left[-\left\{\frac{1}{2} \ln \cos \left(\sqrt{\left.\frac{A}{2} t\right)-\frac{c_{3}^{2}}{4}} \int_{t}^{T} \frac{d t}{\left[\ln \cos \left(\sqrt{\frac{A}{2}} t\right)\right]^{2}+B t+c_{4}}\right\}\right]\right. \\
& \exp \left[-\left\{\frac{\kappa}{\sigma^{2}}(\theta \ln x-x)-\frac{\sqrt{2 A}}{\sigma^{2}} \tan \left(\sqrt{\frac{A}{2}} t\right) x \pm \frac{\sqrt{2} c_{3}}{\sigma \ln \cos \left(\sqrt{\frac{A}{2}} t\right)} \sqrt{x}\right\}\right]
\end{aligned}
$$

\subsection{Reduction to the second Lie canonical form}

Now we focus on the situation when the PDE (4) is equivalent to the second Lie canonical form (14). For this case we have

$$
\sigma^{2} \neq \frac{-8 \kappa \theta \pm 4 \kappa \theta}{-3} \Rightarrow L_{x} \neq 0
$$


Using the same methodology adopted in the previous sections, the transformations that reduce the parabolic equation (4) into the second Lie canonical form are

$$
\begin{aligned}
& f(t)=-\frac{1}{8}\left(\sigma^{2}+\frac{\kappa^{2}}{2}\right), \\
& g(t)=0, \\
& h(t)=\frac{\kappa^{2} \theta}{\sigma^{2}}, \\
& \phi(t)=c_{1} \sqrt{\frac{2}{A}} \tan \left(\sqrt{\frac{A}{2} t}\right), \\
& v(t)=e^{B t+c_{2}} \sqrt{\cos \left(\sqrt{\frac{A}{2}} t\right),}
\end{aligned}
$$

which results in

$$
\begin{aligned}
& \bar{t}=c_{1} \sqrt{\frac{2}{A}} \tan \left(\sqrt{\frac{A}{2}} t\right) \\
& \bar{x}= \pm \frac{2 \sqrt{2} c_{1}}{\sigma \cos \left(\sqrt{\frac{A}{2}} t\right)} \sqrt{x} \\
& \bar{u}=\sqrt{\cos \left(\sqrt{\frac{A}{2}} t\right)}\left(\frac{1}{2} \sigma^{2} x\right)^{-\frac{1}{4}} u \exp \left[B t+c_{2}\right] \\
& \exp \left[\frac{k}{\sigma^{2}}(\theta \ln x-x)-\frac{\sqrt{2 A}}{\sigma^{2}} \tan \left(\sqrt{\frac{A}{2}} t\right) x\right],
\end{aligned}
$$

with $c_{1}, c_{2}, A=-8 f(t)$ and $B=h(t)$ are the constants of integration

\subsection{The fundamental solutions}

We now construct the fundamental solutions when the CIR equation (4) is reduced into the second Lie canonical form. We have deduced the transformations (52) which reduce the PDE (4) to the second Lie canonical form, which is given by

$$
\frac{\partial \bar{u}}{\partial \bar{t}}=\frac{\partial^{2} \bar{u}}{\partial \bar{x}^{2}}+\frac{A}{\bar{x}^{2}} \bar{u}
$$

We first derive the solution for the PDE (43) and then use the transformations (52) to construction of the fundamental solution of the above-mentioned CIR problem.

In order to derive the solution for PDE (53), we make use of the method of separation of variables. We assume the solution of PDE (53) of the form

$$
\bar{u}(\bar{x}, \bar{t})=X(\bar{x}) T(\bar{t}) .
$$

Making use of Eq. (54) into Eq. (43) and separating, we obtain

$$
\begin{array}{r}
\frac{d T}{d \bar{t}}+\lambda T=0, \\
\frac{d^{2} X}{d \bar{x}^{2}}+\left(\frac{A}{\bar{x}^{2}}+\lambda\right) X=0,
\end{array}
$$


where $\lambda \geq 0$ is the separation constant.

Case I: when $\lambda=0$

For this case the general solution of the ODEs (55) and (56) are:

$$
\begin{aligned}
T(\bar{t}) & =K_{1}, \\
X(\bar{x}) & =K_{2} \bar{x}^{\frac{1}{2}(1-\sqrt{1-4 A})}+K_{3} \bar{x}^{-\frac{1}{2}(1-\sqrt{1-4 A})},
\end{aligned}
$$

with $K_{1}, K_{2}$ and $K_{3}$ the constants of integration. Thus the solution of PDE (53) is written as

$$
\bar{u}(\bar{x}, \bar{t})=K_{1}\left[K_{2} \bar{x}^{\frac{1}{2}(1-\sqrt{1-4 A})}+K_{3} \bar{x}^{-\frac{1}{2}(1-\sqrt{1-4 A})}\right] .
$$

In order to find the fundamental solution for the PDE (4) the solution (59) is transformed by means of (52). Making use of Eqs. (59) and (52), the solution $u(x, t)$ is written as

$$
\begin{aligned}
u(x, t)= & K_{1}\left[K_{2} \bar{x}^{\frac{1}{2}(1-\sqrt{1-4 A})}+K_{3} \bar{x}^{-\frac{1}{2}(1-\sqrt{1-4 A})}\right] \\
& \left(\cos \left(\sqrt{\frac{A}{2}} t\right)\right)^{-\frac{1}{2}}\left(\frac{1}{2}\right)^{\frac{1}{4}} \exp \left[-\left\{B t+c_{2}\right\}\right] \\
& \exp \left[-\left\{\frac{k}{\sigma^{2}}(\theta \ln x-x)-\frac{\sqrt{2 A}}{\sigma^{2}} \tan \left(\sqrt{\frac{A}{2}} t\right) x\right\}\right] .
\end{aligned}
$$

Finally, with the substitution of the values of $\bar{t}$ and $\bar{x}$ from (52) into Eq. (60), the solution (60) for the CIR equation (4) takes the form

$$
\begin{aligned}
u(x, t)= & K_{1}\left[K_{2}\left( \pm \frac{2 \sqrt{2} c_{1}}{\sigma \cos \left(\sqrt{\frac{A}{2}} t\right)} \sqrt{x}\right)^{\frac{1}{2}(1-\sqrt{1-4 A})}+K_{3}\left( \pm \frac{2 \sqrt{2} c_{1}}{\sigma \cos \left(\sqrt{\frac{A}{2}} t\right)} \sqrt{x}\right)^{-\frac{1}{2}(1-\sqrt{1-4 A})}\right] \\
& \left(\cos \left(\sqrt{\frac{A}{2}} t\right)\right)^{-\frac{1}{2}}\left(\frac{1}{2} \sigma^{2} x\right)^{\frac{1}{4}} \exp \left[-\left\{B t+c_{2}\right\}\right] \\
& \exp \left[-\left\{\frac{k}{\sigma^{2}}(\theta \ln x-x)-\frac{\sqrt{2 A}}{\sigma^{2}} \tan \left(\sqrt{\frac{A}{2}} t\right) x\right\}\right] .
\end{aligned}
$$

Case II: when $\lambda>0$

For this case the general solution of the ODE (55) is

$$
T(\bar{t})=K_{1} \exp (-\lambda \bar{t})
$$

The general solution of the homogenous equation (56) is (see, e.g., [27])

$$
X(\bar{x})=\left[K_{2} \sqrt{\bar{x}} \mathscr{J}\left(\frac{1}{2} \sqrt{1-4 A} ; \sqrt{\lambda} \bar{x}\right)+K_{3} \sqrt{\bar{x}} \mathscr{Y}\left(\frac{1}{2} \sqrt{1-4 A} ; \sqrt{\lambda} \bar{x}\right)\right],
$$

where $K_{1}, K_{2}$ and $K_{3}$ are the constants of integration and $\mathscr{J}$ and $\mathscr{Y}$ are Bessel functions of the first and second kind, respectively (see, e.g., [28]). Thus the separable solution of the second Lie canonical form (53) is given by

$$
\bar{u}(\bar{x}, \bar{t})=K_{1} \exp (-\lambda \bar{t})\left[K_{2} \sqrt{\bar{x}} \mathscr{J}\left(\frac{1}{2} \sqrt{1-4 A} ; \sqrt{\lambda} \bar{x}\right)+K_{3} \sqrt{\bar{x}} \mathscr{Y}\left(\frac{1}{2} \sqrt{1-4 A} ; \sqrt{\lambda} \bar{x}\right)\right] .
$$


To find the fundamental solution for the PDE (4), the solution (64) is transformed by means of (52). Using Eqs. (64) and (52), the solution $u(x, t)$ is given by

$$
\begin{aligned}
u(x, t) & =K_{1} \exp (-\lambda \bar{t})\left[K_{2} \sqrt{\bar{x}} \mathscr{J}\left(\frac{1}{2} \sqrt{1-4 A} ; \sqrt{\lambda} \bar{x}\right)+K_{3} \sqrt{\bar{x}} \mathscr{Y}\left(\frac{1}{2} \sqrt{1-4 A} ; \sqrt{\lambda} \bar{x}\right)\right] \\
& \left(\cos \left(\sqrt{\frac{A}{2}} t\right)\right)^{-\frac{1}{2}}\left(\frac{1}{2} \sigma^{2} x\right)^{\frac{1}{4}} \exp \left[-\left\{B t+c_{2}\right\}\right] \exp \left[-\left\{\frac{k}{\sigma^{2}}(\theta \ln x-x)-\frac{\sqrt{2 A}}{\sigma^{2}} \tan \left(\sqrt{\frac{A}{2}} t\right) x\right\}\right] .
\end{aligned}
$$

Finally using of $\bar{t}$ and $\bar{x}$ from (52) into Eq. (65), the solution $u(x, t)$ takes the form

$$
\begin{aligned}
u(x, t) & =K_{1} \exp \left(-\lambda c_{1} \sqrt{\frac{2}{A}} \tan \left(\sqrt{\frac{A}{2}} t\right)\right)\left[K_{2} \sqrt{\frac{ \pm \sqrt{2 x} c_{1}}{\sigma \cos \left(\sqrt{\frac{A}{2}} t\right)}} \mathscr{J}\left(\frac{1}{2} \sqrt{1-4 A} ; \sqrt{\lambda} \frac{ \pm 2 \sqrt{2 x} c_{1}}{\sigma \cos \left(\sqrt{\frac{A}{2}} t\right)}\right)\right. \\
& \left.+K_{3} \sqrt{\frac{2 \sqrt{2 x} c_{1}}{\sigma \cos \left(\sqrt{\frac{A}{2}} t\right)}} \mathscr{Y}\left(\frac{1}{2} \sqrt{1-4 A} ; \sqrt{\lambda} \frac{ \pm 2 \sqrt{2 x} c_{1}}{\sigma \cos \left(\sqrt{\frac{A}{2}} t\right)}\right)\right] \\
& \left(\cos \left(\sqrt{\frac{A}{2}} t\right)\right)^{-\frac{1}{2}}\left(\frac{1}{2} \sigma^{2} x\right)^{\frac{1}{4}} \exp \left[-\left\{B t+c_{2}\right\}\right] \exp \left[-\left\{\frac{k}{\sigma^{2}}(\theta \ln x-x)-\frac{\sqrt{2 A}}{\sigma^{2}} \tan \left(\sqrt{\frac{A}{2}} t\right) x\right\}\right] .
\end{aligned}
$$

\subsection{Solution via the Heat Cauchy problem}

In this section, we construct the fundamental solution of the Cauchy problem (4) and (5) for the CIR model. In the previous section, we have found the equivalence transformations which map the PDE (4) to the classical heat equation. We further utilize these transformations for construction of the fundamental solutions of the underlying model.

The transformations are derived in Eq. (46) which reduce the Cauchy problem (4) and (5) for the CIR model into the standard heat Cauchy problem given in Eqs. (34) and (35). The solution to the Cauchy problem (34) and (35) is given by.

$$
\bar{u}(\bar{x}, \bar{t})=\frac{1}{2 \sqrt{\bar{t} \pi}} \int_{-\infty}^{+\infty} \eta(\zeta) \exp \left[-\frac{(\bar{x}-\zeta)^{2}}{4 \bar{t}}\right] d \zeta
$$

for some function $\eta$. We have to transform the solution (67) into the solution of the CIR equation (4). We substitute $\bar{u}(\bar{x}, \bar{t})$ from Eq. (67) into Eq. (46), to obtain

$$
\begin{aligned}
u(x, t) & =\left(\frac{1}{2} \sigma^{2} x\right)^{\frac{1}{4}} \exp \left[-\left\{\frac{1}{2} \ln \cos \left(\sqrt{\frac{A}{2}} t\right)-\frac{c_{3}^{2}}{4} \int_{t}^{T} \frac{d t}{\left[\ln \cos \left(\sqrt{\frac{A}{2}} t\right)\right]^{2}}+B t+c_{4}\right\}\right] \\
& \exp \left[-\left\{\frac{\kappa}{\sigma^{2}}(\theta \ln x-x)-\frac{\sqrt{2 A}}{\sigma^{2}} \tan \left(\sqrt{\frac{A}{2}} t\right) x \pm \frac{\sqrt{2} c_{3}}{\sigma \ln \cos \left(\sqrt{\frac{A}{2}} t\right)} \sqrt{x}\right\}\right] \frac{1}{2 \sqrt{\bar{t} \pi}} \int_{-\infty}^{+\infty} \eta(\zeta) \exp \left[-\frac{(\bar{x}-\zeta)^{2}}{4 \bar{t}}\right] d \zeta .
\end{aligned}
$$


We find $\eta(\zeta)$ in Eq. (68). By keeping in mind the condition (47), we have

$$
\begin{gathered}
\eta(\zeta)=\left(\frac{1}{2} \sigma^{2} x\right)^{-\frac{1}{4}} \exp \left[\frac{1}{2} \ln \cos \left(\sqrt{\frac{A}{2}} T\right)+B T+c_{4}\right] \\
\quad \exp \left\{\frac{\kappa}{\sigma^{2}}(\theta \ln x-x)-\frac{\sqrt{2 A}}{\sigma^{2}} \tan \left(\sqrt{\frac{A}{2}} T\right) x \pm \frac{\sqrt{2} c_{3}}{\sigma \ln \cos \left(\sqrt{\frac{A}{2}} T\right)} \sqrt{x}\right\} .
\end{gathered}
$$

Finally, with the insertion of the values of $\bar{x}$ and $\bar{t}$ from Eq. (46) into Eq. (68), the solution is written as

$$
\begin{aligned}
& u(x, t)=\exp \left[-\left\{\frac{1}{2} \ln \cos \left(\sqrt{\frac{A}{2}} t\right)-\frac{c_{3}^{2}}{4} \int_{t}^{T} \frac{d t}{\left[\ln \cos \left(\sqrt{\frac{A}{2}} t\right)\right]^{2}}+B t+c_{4}\right\}\right] \\
& \exp \left[-\left\{\frac{\kappa}{\sigma^{2}}(\theta \ln x-x)-\frac{\sqrt{2 A}}{\sigma^{2}} \tan \left(\sqrt{\frac{A}{2}} t\right) x \pm \frac{\sqrt{2} c_{3}}{\sigma \ln \cos \left(\sqrt{\frac{A}{2}} t\right)} \sqrt{x}\right\}\right] \\
& \left(\frac{1}{2} \sigma^{2} x\right)^{-\frac{1}{4}} \frac{1}{2 \sqrt{c_{1} \sqrt{\frac{2}{A}} \tan \left(\sqrt{\frac{A}{2}} t\right) \pi}} \\
& \int_{-\infty}^{+\infty} \eta(\zeta) \exp \left[-\frac{\left( \pm \frac{2 \sqrt{2 c_{1}}}{\sigma \cos \left(\sqrt{\frac{A}{2}} t\right)} \sqrt{x}+c_{2} \int_{t}^{T} \frac{d t}{\cos \left(\sqrt{\frac{A}{2}} t\right) \ln \cos \left(\sqrt{\frac{A}{2}} t\right)}+c_{3}-\zeta\right)^{2}}{4 \bar{t}}\right] d \zeta
\end{aligned}
$$

where $\eta(\zeta)$ is defined in Eq. (69). One can deduce the value of the integral in (70) in terms of the Kummer confluent hypergeometric functions and one can also perform the numerical integration.

\section{Concluding remarks}

The class of linear $(1+1)$ parabolic partial differential equations (PDEs) for the Vasicek and Cox-Ingersoll-Ross (CIR) models of financial problems are investigated from the viewpoint of the invariant approach. The classification of these PDEs depend on Lie's classification results of parabolic equations and the use of transformation formulae. This fact substantially simplified the task of obtaining the Cauchy solutions of the models under question. The invariant method enabled us to compute the equivalence transformations which reduced the underlying class of PDEs to the different Lie canonical forms.

For the case of the Vasicek model, we derived the transformations formulae which reduced the Vasicek equation (3) into the first Lie canonical form, which is the heat equation. These transformations are further utilized to obtain the fundamental solutions of the Vasicek equation by employing the well-known fundamental solutions of the heat equation. For the CIR model (4), we have shown that it can be mapped by choosing the appropriate values of the parameters to the first Lie canonical form. Otherwise it is mapped to the second Lie canonical form. For both the cases of the heat equation 
and the second Lie canonical form, we derived the equivalence transformations and obtained the closed-form fundamental solutions of the CIR equation (4).

The use of invariant analysis of parabolic PDEs to obtain the solutions of the corresponding Cauchy problems is significant. Therefore, using the invariant approach, the closed-form solutions of the Cauchy problem for the Vasicek and Cox-Ingersoll-Ross (CIR) models with suitable choice of terminal conditions are derived for the first time. The solution process involved transforming the Cauchy initial value problems into the standard heat Cauchy problem and then taking advantage of the well-known solution to the Cauchy problem of the heat equation.

Also to note here is that invariant criteria utilized can be extended to other parabolic models which transform not only to the heat equation but to other canonical forms as well.

\section{Conflict of Interests}

The authors declare that there is no conflict of interests regarding the publication of this paper.

\section{Acknowledgements}

AB would like to thank the DST-NRF Centre of Excellence in Mathematical and Statistical Sciences (CoE-MaSS), University of the Witwatersrand (WITS), South Africa, for financial support and warm hospitality during the time this research was undertaken. TA would also like to thank CoE-MaSS and University Research Council (URC) of WITS for an enabling postdoctoral fellowship for 2015. FMM thanks the National Research Foundation (NRF) of South Africa for research support through grants.

\section{References}

[1] R.K. Gazizov and N.H. Ibragimov, Lie Symmetry Analysis of Differential Equations in Finance, Nonlinear Dynam. 17(4) (1998) $387-407$.

[2] J. Goard, New Solutions to the Bond-Pricing Equation via Lie's Classical Method, Math. Comput. Model. 32 (2000) 299 - 313.

[3] C.F. Lo and C.H. Hui, Lie-Algebraic Approach for Pricing Moving Barrier Options with Time-Dependent Parameters, J. Math. Anal. Appl. 323 (2006) 1455-1464.

[4] F.M. Mahomed, K.S. Mahomed, R. Naz and E. Momoniat, Invariant Approaches to Equations of Finance, Math. Comput. Appl. $18(3)$ (2013) $244-250$.

[5] A. Bakkaloğlu, T. Aziz, A. Fatima, F. M. Mahomed and Chaudry M. Khalique, Invariant Approach to Optimal InvestmentConsumption Problem: the constant elasticity of variance (CEV) Model, Mathematical Methods in the Applied Sciences, 2016.

[6] C.A. Pooe, F.M. Mahomed and C. Wafo Soh, Fundamental Solutions for Zero-Coupon Bond Pricing Models, Nonlinear Dynam. 36 (2004) $69-76$.

[7] B. İzgi and A. Bakkaloğlu, Fundamental Solution of Bond Pricing in the Ho-Lee Stochastic Interest Rate Model Under the Invariant Criteria, New Trends in Mathematical Sciences, 5, 1, 196-203, 2017.

[8] B. İzgi and A. Bakkaloğlu, Deterministic Solutions of the Stochastic Differential Equations Using Invariant Criteria, Proceedings of ICPAS 2017, 323-326, ISBN: 978-605-9546-02-7, 2017.

[9] B. İzgi and A. Bakkaloğlu, Invariant Approaches for the Analytic Solution of the Stochastic Black-Derman Toy Model ,submitted 2017.

[10] V. Naicker, K. Andriopoulos and P.G.L. Leach, Symmetry Reductions of a Hamilton-Jacobi-Bellman Equation Arising in Financial Mathematics, J. Nonlinear Math. Phys. 12(2) (2005) 268 - 283. 
[11] N.M. Ivanova, C. Sophocleous and P.G.L. Leach, Group Classification of a Class of Equations Arising in Financial Mathematics, J. Math. Anal. Appl. 372 (2010) 273-286.

[12] Y. Liu and Deng-Shan Wang, Symmetry Analysis of the Option Pricing Model with Dividend yield from Financial Markets, Appl. Math. Lett. 24 (2011) 481-486.

[13] N.C. Caister, K.S. Govinder and J.G. O'Hara, Optimal System of Lie group Invariant Solutions for the Asian Option PDE, Math. Meth. Appl. Sci. 34 (2011) 1353-1365.

[14] W. Sinkala, Two ways to Solve, Using Lie Group Analysis, the Fundamental Valuation Equation in the Double-Square-Root Model of the Term Structure, Commun. Nonlinear Sci. Numer. Simulat. 16 (2011) 56-62.

[15] I. Hernández, C. Mateos, J. Núñez and Á.F. Tenorio, Lie Theory: Applications to Problems in Mathematical Finance and Economics, Appl. Math. Comput. 208 (2009) 446- 452.

[16] L. Bachelier, Theorie de la Speculation, Annales Scientifiques de l'Ecole Normale Superieure. 3 (1900) 21 - 86.

[17] R.C. Merton, Optimum Consumption and Portfolio Rules in a Continuous Time Model, J. Economic Theory. 3(4) (1971) 373 - 413.

[18] F. Black and M. Scholes,The Pricing of Options and Corporate Liabilities, J. Political Economy. 81 (1973) 637 - 654.

[19] B.İzgi, Behavioral Classification of Stochastic Differential Equations in Mathematical Finance, Ph.D. Thesis, Istanbul Technical University, 2015.

[20] O. Vasicek, An Equilibrium Characterization of the Term Structure, J. Financial Eco. 5 (1977) 177 - 188.

[21] J.C. Cox, J.E. Ingersoll and S.A. Ross, An Intertemporal General Equilibrium Model of Asset Prices, Econometrica. 53 (1985) $363-384$.

[22] I.K. Johnpillai and F.M. Mahomed, Singular Invariant Equation for the (1+1) Fokker-Planck Equation, J. Physics A: Mathematical and General. 34 (2001) 11033 - 11051.

[23] F.M. Mahomed, Complete Invariant Characterization of Scalar Linear (1+1) Parabolic Equations, J. Nonlinear Math. Phys. 15 (2008) $112-123$.

[24] A.G. Johnpillai, F.M. Mahomed and S. Abbasbandy, Fundamental Solution via Invariant Approach for a Brain Tumor Model and its Extensions, Z. Naturforsch. 69 (2014) 725 - 732.

[25] T.Y.N. Myint-U, Partial Differential Equations of Mathematical Physics, Amercian Elsevier Publishing Company, INC. New York, (1973).

[26] J. Hadamard, Lectures on Cauchy's Problem in Linear Partial Differential Equations, Yale University Press, New Haven, Connecticut, (1923).

[27] A.D. Polyanin and V.F. Zaitsev, Handbook of Exact Solutions for Ordinary Differential Equations, CRC Press, Boca Raton, FL, (1995).

[28] M. Abramowitz and I.A. Stegun, Handbook of Mathematical Functions, New York, (1965). 